\title{
Mechanistic Studies of Cyclin-Dependent Kinase Inhibitor 3 (CDKN3) in Colorectal Cancer
}

\author{
Cheng Yang, Jun-Jun Sun*
}

\begin{abstract}
Colorectal cancer is one of the most severe subtypes of cancer, and has the highest propensity to manifest as metastatic disease. Because of the lack of knowledge of events that correlate with tumor cell migration and invasion, few therapeutic options are available. The current study aimed to explore the mechanism of colorectal cancer in hope of identifying the ideal target for future treatment. We first discovered the pro-tumor effect of a controversial cell cycle regulator, cylin-dependent kinase inhibitor 3 (CDKN3), which is highly expressed in colorectal cancer, and the possible related signaling pathways, by bioinformatics tools. We found that CDKN3 had remarkable effects in suppressing colorectal cancer cell proliferation and migration, inducing cell cycle arrest and apoptosis in a colorectal cancer cell line, SW480 cells. Our study, for the first time, provided consistent evidence showing overexpression of cell cycle regulator CDKN3, in colorectal cancer. The in vitro studies in SW480 cells revealed a unique role of CDKN3 in regulating cellular behavior of colorectal cancer cells, and implied the possibility of targeting CDKN3 as a novel treatment for colorectal cancer.
\end{abstract}

Keywords: CDKN3 - colorectal cancer - apoptosis - cancer cell migration

Asian Pac J Cancer Prev, 16 (3), 965-970

\section{Introduction}

Colorectal cancer is one of the most serve, frequent malignant types of the digestive tract cancer, as the 3rd highest rates of morbidity and mortality in cancerrelated death worldwide (Siegel et al., 2014). Because of the faster pace of lifestyle and globalization of western modernization, the incidence of colorectal cancer continues to rise. In China, in 2010, it was estimated that there were 274,841 new cases diagnosed, with the crude incidence rate of about 2/10,000 (Zheng et al., 2014). Although there is more and more understanding of basic mechanism of developing colorectal cancer, the surgical management remains the mainstream treatment. The genetic cause of colorectal cancer is still remained misery, as neither existent family history nor genetic predisposition was identified (Fearon and Vogelstein, 1990). Recently, there has been significant progress in identifying distinct molecular pathways/markers leading to colorectal cancer. At least 3 pathways have been identified, which are all related with DNA damage. Chromosomal instability pathway is resulted from numerical and structural chromosomal alteration, with loss of heterozygosisty $(\mathrm{LOH})$. Another pathway is the microsatellite instability pathway, which is the results of deregulation of DNA mismatch repair genes, leading to genetic hypermutability. The third pathway in colorectal cancer pathogenesis is known as $\mathrm{CpG}$ island methylation pathway. Notably, the sporadic colorectal cancer frequently exhibits features from all these pathways, suggesting these pathway are not mutually exclusive (reviewed in (Deschoolmeester et al., 2010)). More importantly, all these pathways seem to equally participate in the regulation of many oncogene or tumor repressors, including p53, p21/Cip1/Walf1,p27/ Kip1, E2F and RB1 (Pino and Chung, 2010). Another important factors that have been identified and studied extensively are microRNAs (Orang and Barzegari, 2014), however, targeting microRNAs as a therapeutic option is really hard task.

Despite there are many genetic alternations that contribute to cancer progression, a majority of these mutation are in genes that are involved in regulating cell cycle progression, especially regarding the G1 phase (Sherr, 2000; Ho and Dowdy, 2002). Cylin-dependent kinases (Cdk) and the cylcins with which they interact are major regulators of cell cycle progression. The overall activity of these proteins is dependent on balance of phosphorylation/dephosphorylation events (Sherr and Roberts, 1999). One of the important genes, CDKN3 gene encodes the cdk-assocated protein phosphatase KAP (also known as Cdi1), which dephosphorylates Cdk2 on Thr160 and subsequently activates Cdk2 (Hannon et al., 1994). The finding of CDKN3 being a regulator of cell cycle progression raises the possibility that it might be involved in tumorigenesis. However, reports on the role of CDKN3 in tumors have remained contradictory, or 
Cheng Yang and Junjun Sun*

tissue/cell type specific. In hepatocellular carcinoma and in a hepatoblastoma cell line, aberrant CDKN3 mRNA transcripts that encode protein lacking the activity dephosphorylating Cdk2 (Yeh et al., 2000; Yeh et al., 2003). In contrast, CDKN3 protein was reported to be overexpressed and to enhance tumorigenesis in breast and prostate cancer (Lee et al., 2000). The latter conclusion is difficult to explain, given the inhibitory role of CDKN3 in cell cycle regulation. Thus, the role of CDKN3 in tumorgenesis is still unclear.

Up to now, there is no report about the role of CDKN3 in colorectal cancer. Here, we are the first to employ bioinformatics tools and found the overexpression of CDKN3 in majority of human colorectal cancer, and identified the possible pathways. Then, the in vitro mechanistic studies further showed that inhibition of CDKN3 in a colorectal cancer cell line suppresses cellular proliferation, causes the cell cycle arrest in G1 phase, enhances the apoptotic rates, and regulates the expression of related genes. Importantly, we also found that silencing CDKN3 interfered with the invasion and migration capabilities of these cells, further confirming the role for $\mathrm{CDKN} 3$ as a promoter of malignancy in colorectal cancer.

\section{Materials and Methods}

\section{Materials}

The anti-CDKN3/KAP antibody and anti GAPDH antibody were purchased from Abcam (Cambridge, MA) and Cell Signaling (Beverly, MA), respectively. All other chemicals were obtained either from Sigma or Fisher Scientific (Pittsburgh, PA). All human samples were collected in First Affiliated Hospital of Henan University of Science and Technology (HUST), China. The design and performance of all human sample related experiments were submitted and approved by the approval committee of HUST. CDKN3 siRNA was obtained from Santa Cruz Biotech (Santa Cruz, CA, USA).

\section{Cell culture and treatment}

Human colorectal carcinoma cell lines, including RKO, SW480, SW620, HCT 116 and HT-29 were all purchased from ATCC. All these cells line were maintained in the conditions according to ATCC's instruction, mainly in DMEM medium supplemented with $10 \%$ fetal bovine serum plus antibiotics (100 units penicillin /ml and 100 $\mu \mathrm{g}$ streptomycin $/ \mathrm{ml}$ ). The cells were cultured at $37{ }^{\circ} \mathrm{C}$ under a humidified $5 \% \mathrm{CO}_{2}$ environment.

\section{Cellular proliferation assay}

Cell proliferation assays were performed with a Cell
Counting Kit-8 (Dojindo, Japan). SW480 cells were plated in 24-well plates in triplicate at about $5 \times 10^{4}$ cells per well. Then cells were treated with various treatment conditions and the numbers of cells per well were measured by the absorbance $(450 \mathrm{~nm})$ of reduced WST-8 (2- (2-methoxy4-nitrophenyl)-3- (4-nitrophenyl)-5- (2,4-isulfophen-yl)$2 \mathrm{H}$-tetrazolium, monosodium salt) at the indicated time points.

RNA extraction and quantitative reverse transcriptionPCR (qRT-PCR) analyses

Total RNA from cells was isolated by using Trizol (Invitrogen, Cat\# 15596). Reverse transcription was performed with RevertAid First Strand cDNA Synthesis Kit (Thermo, K1622). Quantitative PCR was performed with SYBR Green PCR Master Mix (Thermo, F-415XL) on Applied Biosystems 7300 Fast Real-Time PCR System. Sequences of primers used are listed as the Table-1. Data were normalized to GAPDH mRNA content, by using efficiency (2- $\Delta \Delta \mathrm{Ct}$ ) method (Livak and Schmittgen, 2001).

\section{Cell cycle flow cytometric analysis}

For FACS analysis, cells were harvested in $0.25 \%$ trypsin solution, fixed in cold $75 \%$ ethanol, and stored at $-20^{\circ} \mathrm{C}$ for 16 hours. Fixed cells were subsequently washed once with cold PBS, treated with RNase A (100 $\mu \mathrm{g} / \mathrm{ml}$ ) in PBS and $50 \mu \mathrm{g} / \mathrm{ml}$ propidium iodide (PI) for 30 min in the dark. DNA content of cells was quantified in a BD Biosciences FACS Calibur with 10,000 events for G0/G1 cell population using BD Biosciences Cell Quest software, and the data were analyzed by FlowJo software (Ashland, OR).

\section{Apoptosis analysis}

The apoptotic cells were detected using annexin V-FITC along with PI solution, by flow cytometry assay according to manufacturer's instruction (Annexin V FITC apoptosis detection kit, Calbiochem). Briefly, cells were treated under various conditions as indicated. Then, cells were gently washed twice with annxin-binding buffer. When cells appear detached from the plate, cells were released with gentle tapping, collected and suspended in cold binding buffer and stained with annexin V FITC and PI solution. Analysis was conducted for 20,000 cells using a flow cytometer with CellQuest software. In each of the graphs, the bottom right quadrant represents cells in early apoptosis.

\section{Transwell invasion assay}

Transwell invasion assay was performed as described

Table 1. Primers for RT-CPR

\begin{tabular}{lcc}
\hline Gene & Forward Primer $\left(5^{\prime}{ }^{\prime}-3^{\prime}\right)$ & Reverse Primer(5' $\left.{ }^{\prime} 3^{\prime}\right)$ \\
\hline CDKN3 & AGCTGCACATCTATCATC & CACTGGTGGTTTCATTTC \\
CDK1 & ACCATACCCATTGACTAAC & ATAAGCACATCCTGAAGAC \\
POLD1 & GCAGGTCAAGGTCGTATC & GAAGGAGCGTGGTGTAAC \\
PCNA & GCCTGACAAATGCTTGCTGAC & TTGAGTGCCTCCAACACCTTC \\
FEN1 & TGTTCTACCGCACCATTC & AGGCTCAGCAGATGTTTG \\
GAPDH & CACCCACTCCTCCACCTTTG & CCACCACCCTGTTGCTGTAG \\
\hline
\end{tabular}


before. Basically, Caki- 2 cells ( 105 cells/transwell) along with indicated treatments (control, scramble or siRNA) were seeded into the upper compartment of invasion chambers. The bottom chambers were filled with normal RPMI 1640 medium. After 24 hours incubation, migrated cells were fixed with $4 \%$ paraformaldehyde and stained with $0.5 \%$ crystal violet in $2 \%$ ethanol. Following termination of the invasion assay, images were obtained under an inverted microscope. Image $\mathrm{J}$ (NIH, Bethesda, MD) was used to count the number of invaded cells (cell counter tool).

\section{SDS-PAGE and western blotting assays}

Cultured cells were collected on ice with $0.5-1 \mathrm{ml}$ of IP buffer and then lysed by sonication for $10 \mathrm{sec}$. Cell lysates were centrifuged for $45 \mathrm{~min}$ at $13,000 \mathrm{~g}$ to remove cellular debris. Total proteins in cell lysates were separated by $10-12 \%$ SDS-PAGE. Western blotting assay was performed by probing with antibodies according to manufacture's instructions. Primary antibodies were detected with horseradish peroxidase-conjugated goat anti-rabbit $\operatorname{IgG}(1: 5,000)$ or anti-mouse $\operatorname{IgG}(1: 10,000)$ using chemiluminescence reagent kit (ECL).

\section{Statistical analysis}

All the data were analyzed using GraphPad Prism 5.04 software (GraphPad Software, La Jolla, CA). Paired Student $t$ test was used for comparison between two groups. Unless otherwise specified in the figure legends, the data are presented as the mean \pm SEM of at least three determinations. Asterisks indicate the degree of significant differences, ${ }^{*} p<0.05,{ }^{* *} p<0.01,{ }^{* * *} p<0.001$.

\section{Results}

CDKN3 is overexpressed in human colorectal cancer

First, we used the TCGA data set to investigate the expression and copy number of CDKN3 in human colorectal cancers. In total, CDKN3 is overexpressed in majority of the colorectal cancers (more than twofold increase, Figure 1A). An independent cohort of 41 normal colon and 262 colon cancer specimen were obtained from the Cancer Genome Atlas (TCGA) network, which is the publicly available cancer database containing the largest genome-wide microarray data from colorectal cancer samples obtained from a multicenter consortium. TCGA colorectal cancer genome-wide microarray data were downloaded from TCGA database online. Our own data also showed the same trend (Figure 1B). We obtained samples from 30 colorectal cancer patients, including 30 tumors samples and 30 adjacent normal tissues. All samples were collected in local hospital, and all protocols were complied with policies and regulatory rules. We found that CDKN3 were overexpressed in tumor tissues, significantly higher than in normal tissues (upper panel). In addition, the ratio of CDKN3 expression level in tumor tissues to normal tissues for the same patients is very close to 2 (lower panel).

Next, we used gene set enrichment analysis (GSEA) to identify the enriched gene sets in normal tissue group and colorectal cancer group. The gene sets were based on published papers or from the Molecular Signature Database (MsigDB, http://www.broadinstitute.org/gsea/ $\mathrm{msigdb} /$ ). These gene sets were obtained from multiple sources including online pathway databases, biochemical literature, and mammalian microarray studies. The values for each gene set were used to rank the functional representation of the significant genes in each gene set by their significance to the list of targets, thereby identifying biological processes likely to be affected. As expected, because of the identification of role of CDKN3 in colorectal cancer, a cell cycle regulator signature is enriched in colorectal cancer group (Figure 1C). Meanwhile, the other enriched gene set was identified as cellular DNA replication set (Figure 1D). Both analysis data suggested that CDKN3 might play an important role in tumorigenesis of colorectal cancer.

CDKN3 highly expressed in SW480 and Inhibition of CDKN3 Suppresses the Proliferation of Colorectal Cancer Cells

To better understand the role of CDKN3 in colorectal cancer development, we first sought to examine the protein expression of CDKN3 in multiple colorectal cell lines. RKO, SW610, SW480, HCT116, HT-29, the cell lines derived from different human colorectal adenocarcinoma, were used here. SW480 showed the highest expression level of CDKN3, among all colorectal cancer cells lines (Figure 2A). We then knock-down CDKN3 in this cell line, by using CDKN3 siRNA. qRT-PCR analysis showed that there was about $60 \%$ reduction in CDKN3 mRNA expression after applying CDKN3 siRNA. Western blotting assay confirmed the efficiency of this siRNA in
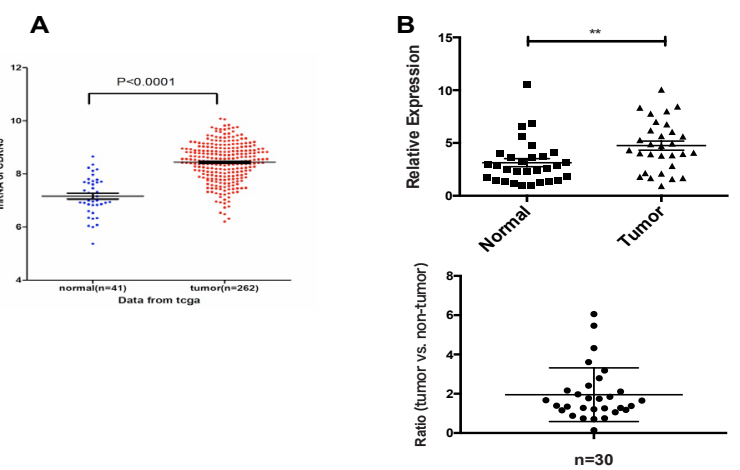

C

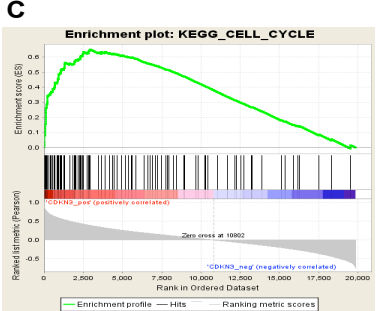

D

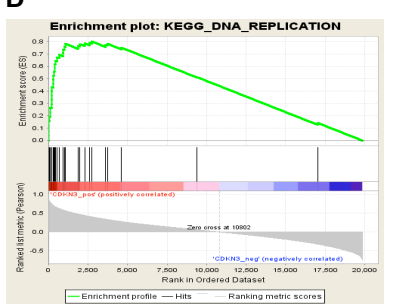

Figure 1. CDKN3 is Overexpressed in Human Colorectal Cancer. A). In Cancer Genome Atlas (TCGA) database, CDKN3 was up regulated in human colorectal cancer, as compared to normal colon tissues. B). CDKN3 was up-regulated in our own study, including 30 colorectal cancer samples and normal colon tissues from patients in local hospital. Gene set enrichment analysis (GSEA) showed that the role of CDKN3 in colorectal cancer was mostly related with cell cycle regulation $\mathbf{C}$ ) and DNA replication $\mathbf{D}$ ) 
Cheng Yang and Junjun Sun*

SW480 cells (Figure 2C). To study if silencing CDKN3 has any effects on cellular proliferation rates, we measure the cell proliferation in presence of scramble or CDKN3 siRNA in SW480 cells. After 24 hours transfection with siRNA, the inhibition of CDKN3 significant suppressed the proliferation rate of SW480 cells in the presence of CDKNs siRNA when compare to non siRNA or scramble siRNA, measured by WST-8 assay. The difference was even bigger when incubation time reached 72 hours, suggestion that CDKN3 really played a role in mediating colorectal cell proliferation. POLD1 (DNA polymerase delta catalytic subunit) and FEN1 (flap endonuclease-1) are two important protein involved in DNA replication. The mRNA levels of both factors were down regulated by the inhibition of CDKN3, further confirming that CDKN3 played a stimulatory role in regulating colorectal cancer cell proliferation.

Slicing CDKN3 causes the cell cycle arrest and promotes apoptosis

As cell cycle is normally linked to cellular proliferation, to further understand the mechanism that the inhibitory effects of knocking-down CDKN3 on cancer cell
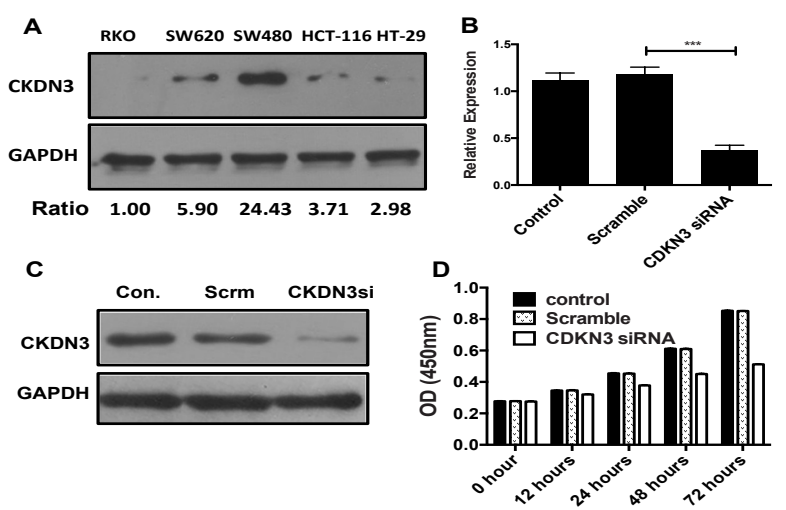

E
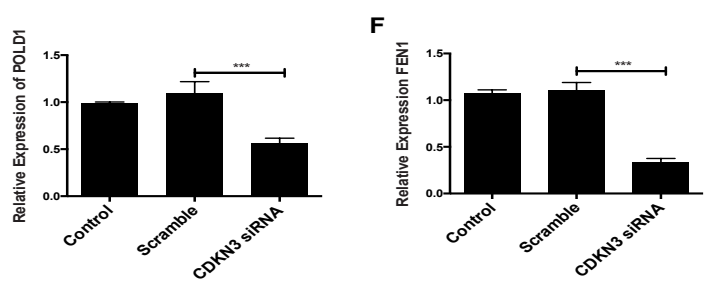

Figure 2. Inhibition of CDKN3 Suppresses the Proliferation of Colorectal Cancer Cells, SW480. A) CDKN3 was highly expressed in SW480, among all other cell lines derived from different human colorectal adenocarcinoma. B\&C) CDKN3 siRNA significantly down-regualtes the mRNA expression B) and protein expression of CDKN3 C). The intensity of bands was determined by Image $\mathbf{J}$, and ratio of CDKN3 to GAPDH were shown. D). Suppressing of CDKN3 aggravates cell proliferation of SW480 cells. Cell proliferation was determined by measuring reduced WST- 8 at $450 \mathrm{~nm}$, at 0 , 24, 48 and 72 hours respectively, after applying CDKN3 siRNA. E\&F) Inhibition of CDKN3 in SW480 leads to the down-regluation of mRNA levels of POLD1 E) and FEN1 F). The data were analyzed by Student $t$ test, and were presented as the means \pm s.e.m. $(\mathrm{n}=3-5),{ }^{*}, p<0.05$; $* *, p<0.01 * * *, p<0.001$ proliferation, we investigated to see if cell cycle progression was affected by alteration in CDKN3 gene. Give the role of CDKN3 in cell cycle regulation (Sherr and Roberts, 1999), as expected, compared to controls and scramble siRNA group, SW480 cells treated with CDKN3 siRNA had a significantly increased cell population in G1 phase, less cells in $\mathrm{S}$ phase (Figure 3A), indicating a G1/S cell cycle arrest. Applying both Snail shRNA and quercetin, more cells were found in G1 and much less cells were in $\mathrm{S}$ phase, as compared to either Snail shRNA or quercetin treatment alone. In addition, important genes involved in cell cycle progression, such as CDKN1 and PCNA, were significantly down regulated after slicing CDKN3 in SW480 cells (Figure 3B\&C), measured by qRT-PCR. This observation implied that suppressing colorectal cancer cell proliferation by CDKN3 siRNA is very like through inhibiting cell cycle progress.

Cellular proliferation, differentiation and death are the basic and essential processes in multicellular organisms. Especially, in cancer cells, uncontrolled proliferation can sometime be associated with a high level of apoptosis, suggesting that cell proliferation and apoptosis pathways are closely linked (Evan and Vousden, 2001). To further

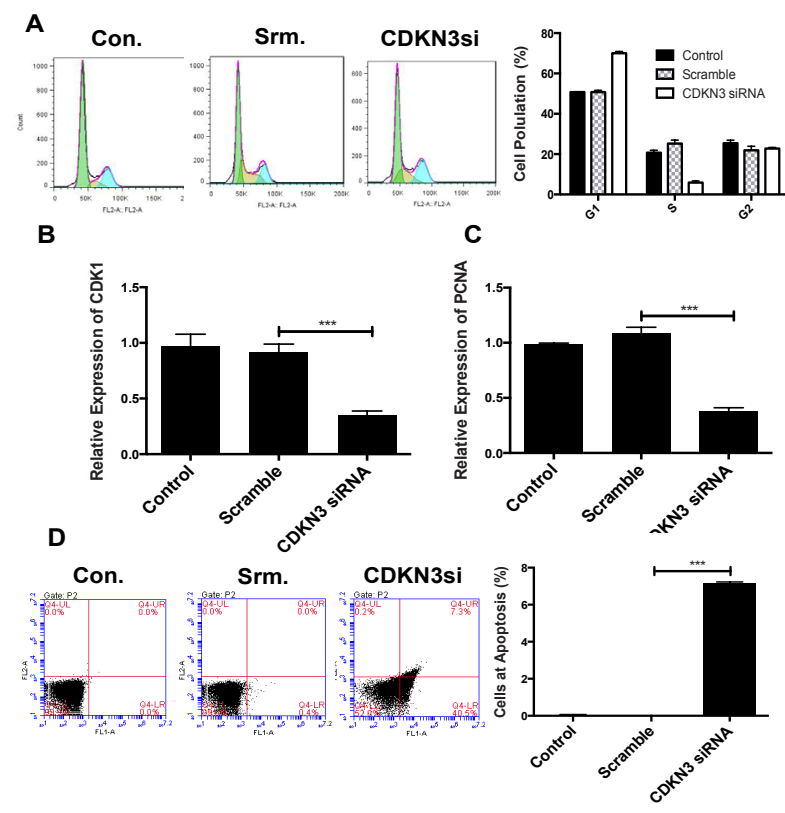

Figure 3. Slicing CDKN3 Leads to G1/S Phase Cell Cycle Arrest and Induces Apoptosis in SW480 Cells. A). $\mathrm{CDKN} 3$ siRNA causes G1/S cell cycle arrest. Cells were then collected by trypsinization and stained with PI solution prior to subject to FACS analysis. Raw data were processed by FlowJo software, and expressed as the percentage of cells population in certain cell cycle. (B\&C). CDKN3 siRNA down-regulates mRNA levels of CDK1 and PCNA, measured by quantitative real time PCR. The gene expression levels were normalized to GAPDH and control group. The data were analyzed and presented as the means \pm s.e.m. $(\mathrm{n}=3-5),{ }^{*} p<0.05$; $* *, p<0.01$ $* * *, p<0.001$. (D) CDKN3 siRNA causes apoptosis. Cells were then harvested and co-stained with FITC-conjugated annexin $\mathrm{V}$ and PI for 15 minutes, and analyzed by flow cytometer as described in Methods. Histogram represents mean \pm SE of percentage of cell population in apoptotic phase in three independent experiments. Significant difference from control cells is indicated by $* p<0.05$ and $* * p<0.01$ 
access the pro-tumorgenic features of CDKN3, we sought to find out if CDKN3 siRNA has any effects on cellular apoptosis. We utilized Annexin-V staining, which detects phosphatidylserine (PS) when it is flipped and translocated from the inner to the outer leaflet of the membrane in the intermediate stages of apoptosis. Highly fluorescent annexin $\mathrm{V}$ conjugates provide quick and reliable detection methods for studying apoptosis. There were nearly no apoptotic cells that can be detected in parental SW480 cells or cells treated with scramble siRNA. While, CDKN3 siRNA could largely enhance apoptotic cell population by about $7 \%$ (Figure 3C). These data provide an inverse relationship between proliferation and apoptosis in colorectal cancer cells treated by CDKN3 siRNA, and partially explains that suppressed cellular proliferation by CDKN3 siRNA is very likely due to enhanced apoptosis.

CDKN3 siRNA suppresses the invasion and migration capabilities in colorectal cancer cell line

Migration and invasion of colorectal cancer cells are essential processes during colorectal carcinoma metastasis. To evaluate the invasion capability in vitro of CDKN3 siRNA on SW480 cells, we examined the effect of CDKN3 siRNA on the invasion Transwell assay. The data suggested that CDKN3 siRNA significantly abolished the invasion and migration abilities of Caki-2 cells (Figure 4A). The average numbers of cells invaded into the
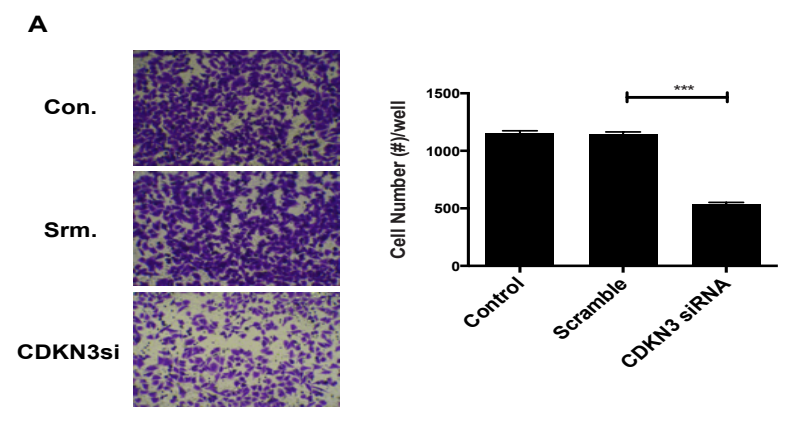

B

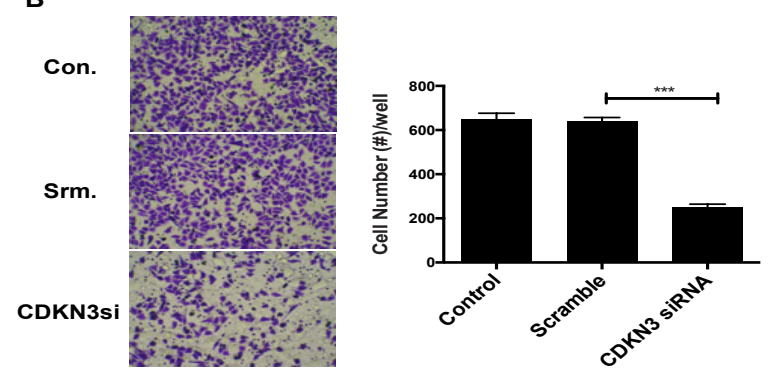

Figure 4. Inhibition of CDKN3 Suppresses SW480 Cell

Invasion and Migration. A). Trans-well assay showed that CDKN3 siRNA inhibits SW480 cells were first plated in the top compartment of invasion chambers. After siRNA transfecton, migrated cells were then fixed and stained with crystal violet. Then, the images of cells in bottom chambers were taken under an inverted microscope (right panels). Cells numbers were counted by Image J. Histogram (left panel) represents mean \pm $\mathrm{SE}$ of three independent experiments. Significant difference from control cells is indicated by $* p<0.05$ and $* * p<0.01$. B). Invasion assay were performed using basement membrane coated by Matrigel. The images taken and quantification were described in trans-well assay lower chamber was 533 in presence of CDKN3 siRNA, compared to 1152 cells in control group and 1146 cells in scramble siRNA group. Tumor cell invasion assay was also employed here to determine the invasive phenotype of tumor cells. Cells with higher invasive ability are able to across the basement membrane coated by Matrigel. Our data here showed again that much less of cells were able to cross the membrane and invade to the lower chamber (Figure 4B). Our results strong suggested that knocking down of CDKN3 siRNA treatment resulted in the suppression of the migration and invasive capabilities in SW480 cells. These data correlated the fact that CDKN3 siRNA inhibited the cellular proliferation and promoted apoptosis in SW480 cells.

\section{Discussion}

Cell cycle regulation is suggested to be the driving force of typical tumorigenesis. "Sustaining Proliferative Signaling" was listed as one of six hallmarks of cancer, in a comprehensive review article published a few years ago by Hanahan and Weinberg (Hanahan and Weinberg, 2011). Therefore, cell cycle regulators are often used as potential therapeutic targets (Tamgue et al., 2013). CDKN3 was initially thought to be an inhibitor for cell cycle progression (Poon and Hunter, 1995), while the latest studies challenge this idea and thus the role of CDKN3 in carcinogenesis has remained a puzzle. Overexpression of CDKN3 has been associated with breast cancer, prostate cancer, cell carcinoma, ovarian cancer and hepatocellular carcinoma. Consistent with these previous reports, we here also found that CDKN3 was overexpressed in colorectal cancer, in a data set in TCGA database with 262 colon cancer specimen, as well as in 30 colon cancer samples collected in our local hospital. The mechanism of CDKN3 higher cancer-associated expression has not been fully unveiled yet, but may be related with hypomethylation on its promoter region (Niculescu et al., 2004). To better understand this pro-tumorigenetic function of CDKN3, we first employed bioinformatics tool to postulate that CDKN3 might regulate the tumorigenesis by regulating the genes expression in DNA replication and cell cycle progression. To start the detailed mechanistic study, we first compared the expression level of CDKN3 in the multiple colorectal cancer cell lines, and identified SW480 cell line has the highest CDKN3 expression level. SW480 cell line then was used in all following in vitro experiments.

The role of CDKN3 on cell proliferation has been well studied, but controversial. CDKN3 has been shown to prevent the induction of $\mathrm{p} 21$ and thereby inhibit cell cycle progression, however its pro-proliferation role has been shown in many cancer types, such as breast cancer, ovarian cancer and etc. In case of colorectal cancer, we found that knock-down of CDKN3 cause a significant reduction in cellular proliferation in SW480 cell line. In keeping with this observation, two DNA replication associated genes, POLD1 and FEN1, were shown to be highly down-regulated in cells transfected with CDKN3 siRNA. Cellular proliferation is closely related with cell cycle progression and apoptosis rates. The reduction 
Cheng Yang and Junjun Sun*

in cellular proliferation could results from cell cycle arrest, and/or due to advanced cell death or apoptosis. As expected, CDKN3 siRNA caused significant cell cycle arrest at G1/S phase. In addition, other pro-cell cycle regulators, such as CDKN1 and PCNA, were both downregulated by $\mathrm{CDKN} 3$, supporting the inhibitory role of CDKN3 siRNA in colorectal cancer. Meanwhile, Annexin V/PI staining showed that CDKN3 siRNA leaded to $7 \%$ of total cells undergoing apoptosis, v.s. nearly $0 \%$ of cells in apoptosis in control groups. Therefore, we can conclude here that $\mathrm{CDKN} 3$ played a stimulatory/promoting role in colorectal cancer cell proliferation, associated with cell cycle progression and prevention of apoptosis.

In various tumors, tumorigenesis, invasion and metastasis are forming a complicated network, involving many molecular signaling pathways, cellular factors and other tumor-promoting factors. Particularly in colorectal cancer, tumor invasion and metastasis, in addition to the biological activity of colon cell transformation and migration, currently receive remarkable attention. The treatment of colorectal cancer is becoming extremely difficulty, as metastatic colon cancer is more common in patients now days (Deschoolmeester et al., 2010; Siegel et al., 2014). Thus, investigating colorectal cancer metastasis and infiltration are becoming focal points in colorectal cancer research. The role of CDKN3 on cellular metastasis is rarely reported and inspired us to further investigate whether CDKN3 is of any relationship with cellular invasion. Here, in our research, in specific colorectal cancer cell line $\mathrm{S} 480$, we first provided the consistent data showing that CDKN3 siRNA was able to suppress the invasive ability of SW480 cells in a transwell and cellular invasion assay, indicating that $\mathrm{CDKN} 3$ could really affect the metastasis potential of colorectal cancer cells. To our knowledge, this is the first report of CDKN3 on tumor metastasis other than its pro-proliferation functions.

In summary, we demonstrated that promotable role of CDKN3 in colorectal cancer cellular proliferation, associated with cell cycle progression and apoptosis, by examining the effects of CDKN3 siRNA on SW480 cell line. Our findings provide new and first insights into the molecular mechanisms of CDKN3-mediated proliferation, cell cycle regulation and apoptosis, suggesting that the targeting CDKN3 could be a novel therapeutic strategy for the prevention and treatment of human colorectal cancer.

\section{Acknowledgements}

We thank people at Sun's laboratory for technical supports and critical reading of the manuscript. This work was supported by grants from First Affiliated Hospital of Henan University of Science and Technology, Luoyang, China.

\section{References}

Deschoolmeester V, Baay M, Specenier P, et al (2010). A review of the most promising biomarkers in colorectal cancer: one step closer to targeted therapy. Oncologist, 15, 699-731.

Evan GI, Vousden KH (2001). Proliferation, cell cycle and apoptosis in cancer. Nature, 411, 342-8.
Fearon ER, Vogelstein B (1990). A genetic model for colorectal tumorigenesis. Cell, 61, 759-67.

Hanahan D, Weinberg RA (2011). Hallmarks of cancer: the next generation. Cell, 144, 646-74.

Hannon GJ, Casso D, Beach D (1994). KAP: a dual specificity phosphatase that interacts with cyclin-dependent kinases. Proc Natl Acad Sci USA, 91, 1731-5.

Ho A, Dowdy SF (2002). Regulation of G (1) cell-cycle progression by oncogenes and tumor suppressor genes. Curr Opin Genet Dev, 12, 47-52.

Lee SW, Reimer CL, Fang L, et al (2000). Overexpression of kinase-associated phosphatase (KAP) in breast and prostate cancer and inhibition of the transformed phenotype by antisense KAP expression. Mol Cell Biol, 20, 1723-32.

Livak KJ, Schmittgen TD (2001). Analysis of relative gene expression data using real-time quantitative PCR and the 2 (-Delta Delta C (T)) Method. Methods, 25, 402-8.

Niculescu MD, Yamamuro Y, Zeisel SH (2004). Choline availability modulates human neuroblastoma cell proliferation and alters the methylation of the promoter region of the cyclin-dependent kinase inhibitor 3 gene. $J$ Neurochem, 89, 1252-9.

Orang AV, Barzegari A (2014). MicroRNAs in colorectal cancer: from diagnosis to targeted therapy. Asian Pac J Cancer Prev, 15, 6989-99.

Pino MS, Chung DC (2010). The chromosomal instability pathway in colon cancer. Gastroenterology, 138, 2059-72.

Poon RY, Hunter T (1995). Dephosphorylation of Cdk2 Thr 160 by the cyclin-dependent kinase-interacting phosphatase KAP in the absence of cyclin. Science, 270, 90-3.

Sherr CJ (2000). Cell cycle control and cancer. Harvey Lect, 96, 73-92.

Sherr CJ, Roberts JM (1999). CDK inhibitors: positive and negative regulators of G1-phase progression. Genes Dev, 13, 1501-12.

Siegel R, Desantis C, Jemal A (2014). Colorectal cancer statistics, 2014. CA Cancer J Clin, 64, 104-17.

Tamgue O, Chai CS, Hao L, et al (2013). Triptolide inhibits histone methyltransferase EZH2 and modulates the expression of its target genes in prostate cancer cells. Asian Pac J Cancer Prev, 14, 5663-9.

Yeh CT, Lu SC, Chao CH, et al (2003). Abolishment of the interaction between cyclin-dependent kinase 2 and Cdkassociated protein phosphatase by a truncated KAP mutant. Biochem Biophys Res Commun, 305, 311-4.

Yeh CT, Lu SC, Chen TC, et al (2000). Aberrant transcripts of the cyclin-dependent kinase-associated protein phosphatase in hepatocellular carcinoma. Cancer Res, 60, 4697-700.

Zheng ZX, Zheng RS, Zhang SW, et al (2014). Colorectal cancer incidence and mortality in China, 2010. Asian Pac J Cancer Prev, 15, 8455-60. 